\title{
DESIGN OF COMPLIANT MECHANISMS FOR ENGINERING SUPPORT - A TOPOLOGY OPTIMISATION APPROACH
}

\section{Dr.G.Arunkumar ${ }^{1}$ and Mr.A.Pandiyan ${ }^{2}$}

\#1 Professor and Head, Department of Mechanical Engineering, Sathyabama University, Chennai - 602105, India, Phone No: +91 9894608969

\#2 Associate Professor, Department of Mechanical Engineering, Saveetha University, Chennai 602 105, India, Phone No: +91 98894970549

\section{ABSTRACT}

Compliant mechanisms are the focus of active research because of the flexibility, stability and unitized construction. It is a single elastic continuum used to transfer the force and motion mechanically by elastic deformation without any links and joints. Authors proposed a topology optimisation method for designing a compliant amplifier and steps for conducting the numerical experiments for any design domains with different types of constraint. The paper narrates the applications of the compliant mechanisms in new age industries like automotive, aerospace and other service industries for engineering support with specific examples. Numerical experiments are carried out for different basic configurations to design the compliant amplifier. The compliant amplifier is used for amplifying the displacement and stroke performance of the strain actuators when integrated with Piezo actuators. The objective is to maximize the geometric advantage of the compliant amplifier. The maximization of the objective is accomplished efficient design of compliant mechanisms by the topology optimisation approach. The analysis results help to select the best compliant amplifier design from the basic configurations. The outcome of work will be useful for all types of strain Actuators.

Keywords: Compliant Mechanisms, Geometric Advantage, Topology Optimisation, Numerical Experiments

\section{Corresponding Author: Arunkumar G}

\section{INTRODUCTION TO COMPLIANT MECHANISM}

Mechanism is a mechanical device used to transfer the force, motion or energy from input to output. But compliant mechanism is a flexible structure that elastically deforms without joints to produce a desired force or displacement at the output [1]. Unlike rigid-body mechanisms, compliant mechanisms gain some of their mobility from the deflection of flexible members rather than from movable joints only $[1,2]$. 


\subsection{Compliant Mechanism and Nature}

Humans and nature often have differing philosophies on mechanical design. Stiff structures are usually preferred by humans because for many, stiffness means strength. Devices that must be capable of motion are constructed of multiple stiff structures assembled in such a manner as to allow motion (e.g., door hinges, linkages and roller bearings). However, stiffness and strength cannot be equated. Stiffness is a measure of how much something deflects under load, whereas strength is how much load can be endured before failure [3]. Despite of human tendencies, it is possible to make things that are flexible and strong. Nature used stiff structures where needed (tree trunks, bones, teeth, and claws) but in living organisms, it more often relies on flexibility in living organisms. bee wings, bird wings, tree branches, leaf stems, fish and single-celled organisms are only a few examples of creations that use compliance to their advantage. Nature also has advantage of growing living things, and no assembly is required [1, 4].

The contrast between nature and human design is easily identifiable when human try to replace one of the nature's products. For example, a human heart valve is a compliant one-way valve that is capable of sustaining billions of cycles without failures [4]. However, most of the artificial heart valves use a number of assembled stiff parts with pin joints to obtain motion. They also have a comparatively short life, cause difficulty in blood flow and often damage blood cells.

\subsection{Advantages of Compliant Mechanisms}

Compliant mechanisms may be considered for use in a particular application for a variety of reasons. The advantages of compliant mechanisms are considered in two categories: cost reduction (part-count reduction, reduced assembly time and simplified manufacturing processes) and increased performance (increased precision, increased reliability, reduced wear, reduced weight, and reduced maintenance) [6]. An advantage of compliant mechanisms is the potential for a dramatic reduction in the total number of parts required to accomplish a specified task some mechanisms may be manufactured from an injection-moldable material and be constructed of one piece $[5,6]$.

\section{DESIGN ENGINEERING SUPPORT}

The engineering support for compliant design and validation (by topology optimisation) is to a great extent assisted by computer simulations of compliant mechanism response subjected to real life loading conditions [7]. Computer simulations are an excellent means of understanding the complex mechanical behaviour and different topology of the domain using the visualization and graphic capabilities available with FE tools such as Ansys, Nastran, etc [8].

\subsection{Topology Optimisation}

It is a material distribution method. The method used to find the best use of material for a body/domain. Also to find the optimum shape and size of a linearly elastic structure. The topology optimization method can be used to design compliant mechanical amplifiers with any direction of force and motion transmission $[9,10]$. The compliant mechanical amplifiers are the compliant mechanisms which are used to amplify the force and displacement at the output $[11,12]$. 
One type of smart material actuator typically used in adaptive structures is an induced-strain piezo-ceramic stack actuator. Piezoceramic (PZT) actuators are solid-state devices, which offer the advantages of high energy density and high output force when compared with conventional methods of actuation [13]. One limitation of PZT actuators, however, is that they are capable of producing only 0.1 percent strain, resulting in a restricted range of motion. Thus limiting the stroke Performance. To increase the effective stroke of the actuator, compliant amplifiers have been designed to provide mechanical amplification. However it can be applicable for the strain actuators like piezoceramic actuators, and piezoelectric actuators $(7,13]$. Designers of these mechanical amplifiers typically employ a single-piece compliant amplifier as a coupling structure with the PZT actuator to avoid problems associated with clearances and backlash in hinge joints [14]. Numerical Experiments on Compliant mechanisms (made by polypropylene) Integrated with strain actuator has been considered for displacement amplification of the actuators using topology optimisation method.

\subsection{Geometrical Advantage}

The Geometrical advantage and the procedure to obtain maximum geometrical advantage from a compliant mechanism or amplifier. The force provided by the stack actuator to the compliant amplifier is modeled as a point load at 'a' (Figure 2). This approach is not restricted to symmetric problems however it can be applied to any shape and size design domain with any set of loading and geometrical constraints [6]. Also the magnitude of the force provided by the stack is proportional to the voltage applied to it and the direction is along the longitudinal strain $[8,15]$.

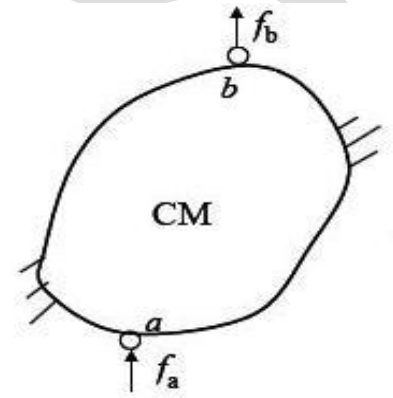

Figure 2 Compliant mechanism with unconstraint output

Max (GA) is the ratio of the output displacement of actuator at ' $b$ ' to the displacement at the point ' $a$ '.

$$
\max (\mathrm{GA})=\frac{\Delta_{\text {out }}}{\Delta_{\text {in }}}=\frac{\mathrm{V}^{\mathrm{T}} \mathrm{KU}}{\mathrm{U}^{\mathrm{T}} \mathrm{KU}}
$$

Constraint equations are given as

Subjected to,

$$
\begin{aligned}
\mathrm{F}_{\text {in }} & =\mathrm{KU}, \\
\mathrm{F}_{\mathrm{d}} & =\mathrm{KV}, \\
\text { Volume } & \leq \mathrm{V}_{\mathrm{o}}
\end{aligned}
$$

Where $\mathrm{K}$ is the global stiffness matrix, $\mathrm{U}$ the deflection vector at ' $\mathrm{a}$ ', and $\mathrm{V}$ the deflection vector at ' $b$ '. The output displacement is equal to mutual stain energy. 
The Design of Compliant Amplifier originated from basic design geometry or domain.

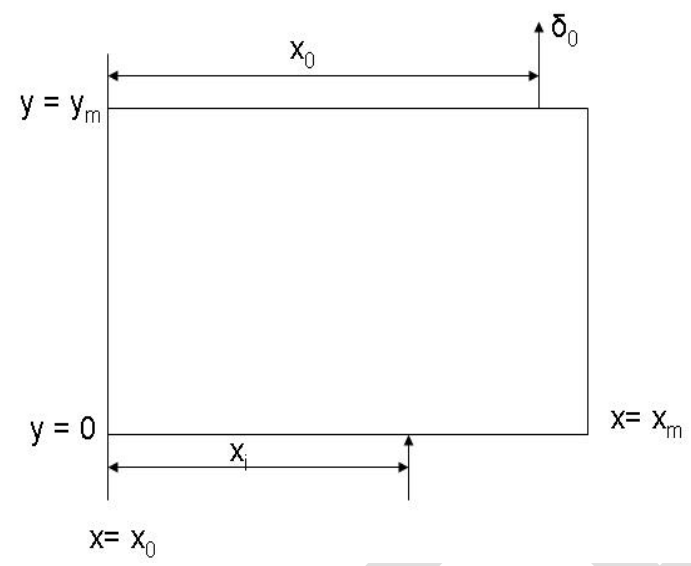

$\mathbf{F}_{\mathbf{i}}$

Figure 3 Basic Design domain for Compliant Amplifier

Three types of configurations (Rectangular, taper, Hexogonal domain) with point constraints (all corners of the respective domain is fixed) has been taken for analysis is shown in figure 4.

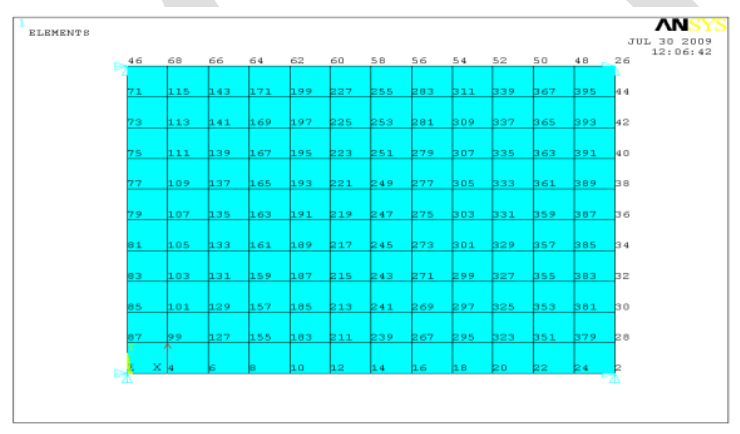

a) Rectangular domain with point constraints

Three types of configurations (Rectangular, taper, hexogonal domain) with surface constraints (left and right end of the surface is fixed) has been taken for analysis is shown in figure 5 .

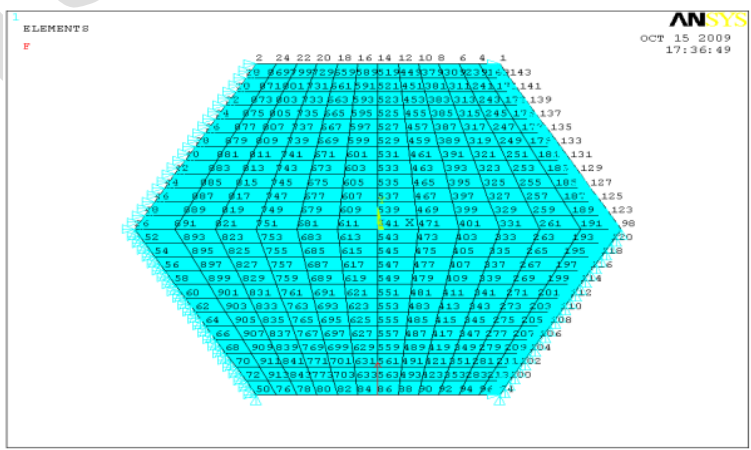

Hexogonal domain with surface constraints

Figure 5 Geometry taken for analysis with surface constraints 
DOI : https://dx.doi.org/10.26808/rs.st.i7v5.10

International Journal of Advanced Scientific and Technical Research

\subsection{Topology Optimisation of Rectangular and Taper domain with Point Constraints}

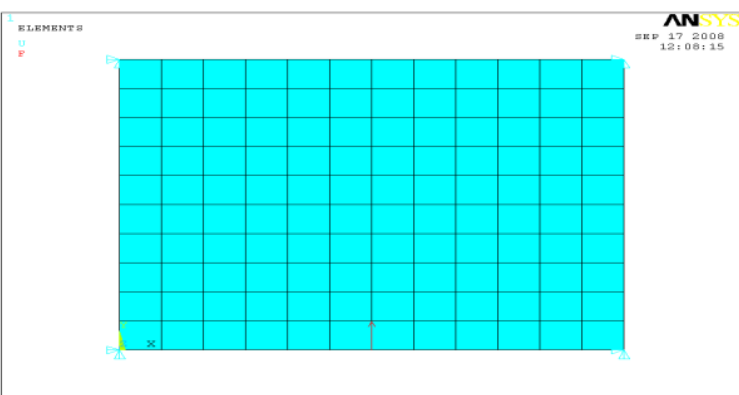

Figure 6 (a) Domain with force applied at input point

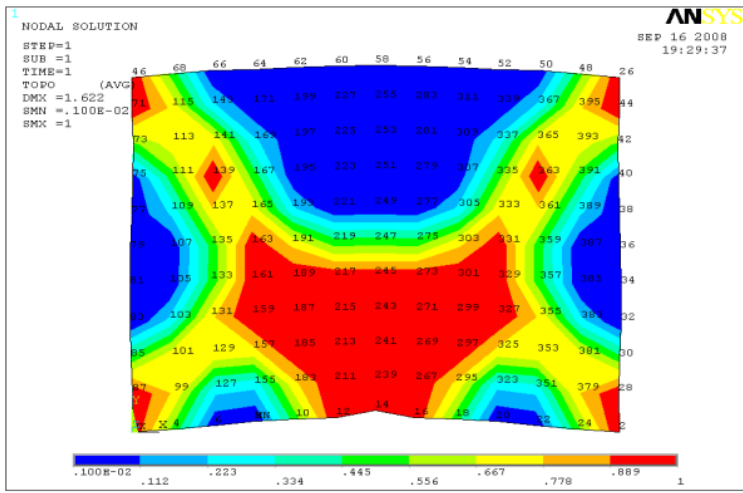

(b) Converged solution after topologically optimized

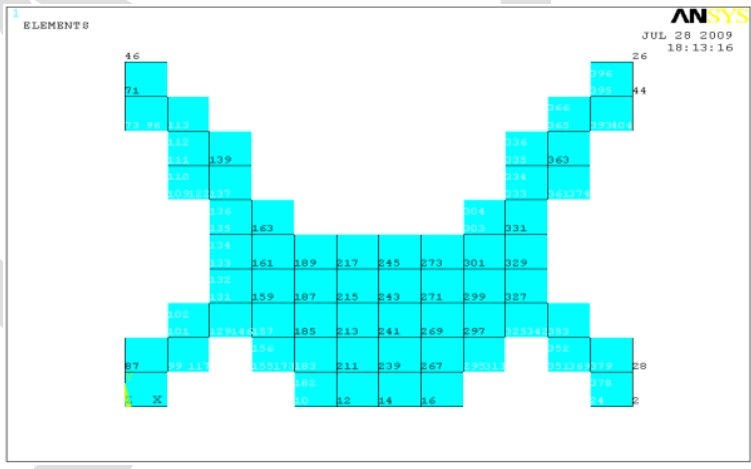

(c) Topologically optimised domain

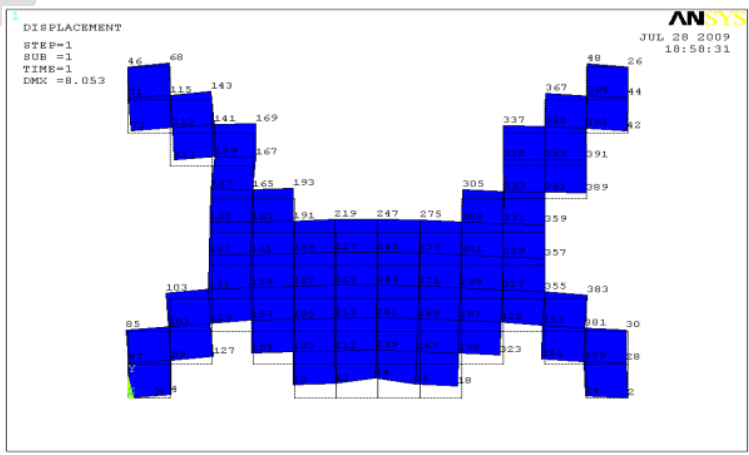

(d) Displacement plot 


\section{ANALYSIS OF GEOMETRY WITH POINT CONSTRAINTS}

\subsection{Effect of Input Location $\left(\mathbf{X}_{\mathbf{i}}\right)$}

To study the effect of input location, input force $\left(\mathrm{F}_{\mathrm{i}}\right)=300 \mathrm{~N}$, output location $\left(\mathrm{X}_{0}\right)=\mathrm{x}_{0} / \mathrm{x}_{\mathrm{m}}$ $=0.5$ are kept constant. Input force is applied at various input locations $\left(\mathrm{X}_{\mathrm{i}}=\mathrm{x}_{\mathrm{i}} / \mathrm{x}_{\mathrm{m}}\right)$ and the results are shown in Figure 8, and Figure 9.

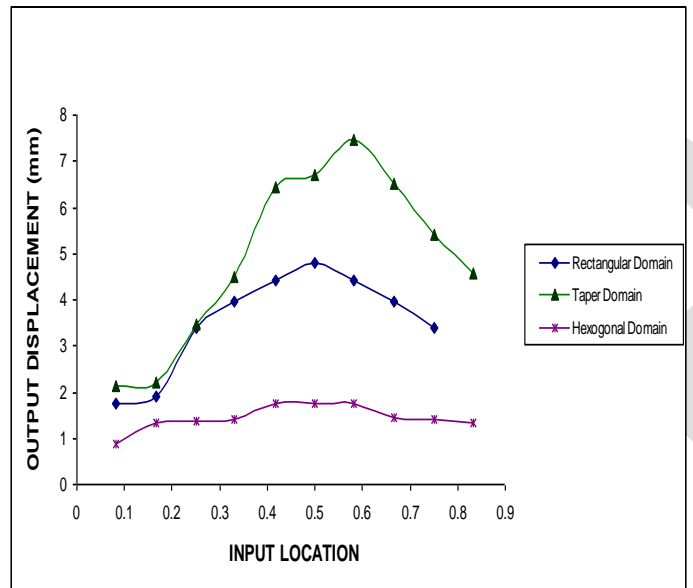

\section{Figure 8 Effect of $\left(X_{i}\right)$ on output displacement}

From Figure 8 the maximum output displacement is obtained at $\left(\mathrm{X}_{0}=\mathrm{x}_{0} / \mathrm{x}_{\mathrm{m}}=0.5\right)$ is $7.48 \mathrm{~mm}$.

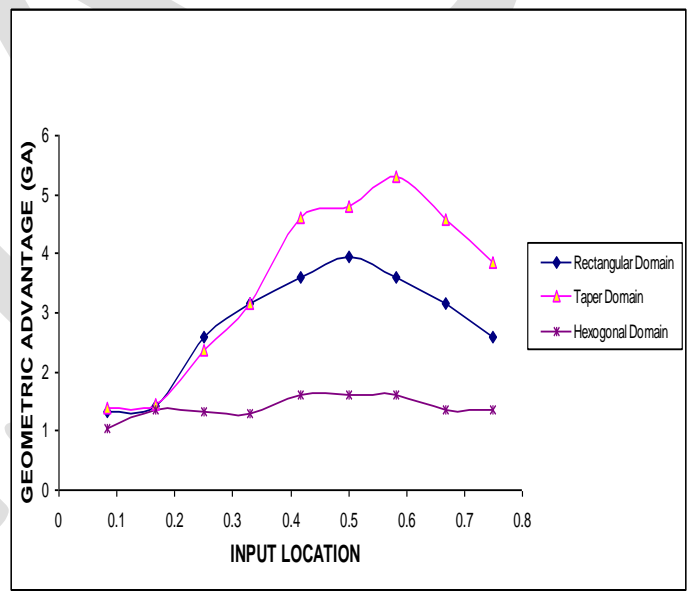

Figure 9 Effect of $\left(X_{i}\right)$ for geometrical advantage

From the Figure 9 the geometric advantage will be maximum at the input location $\mathrm{X}_{\mathrm{i}}=$ $\left(\mathrm{x}_{\mathrm{i}}\right) /\left(\mathrm{x}_{\mathrm{m}}\right)=0.55$ and the corresponding value is 5.32 .

When the input location is varied from the left end to the middle, the output displacement continuously increases and reaches a maximum around the midspan and then decreases towards the right end for all the three geometries. The configuration at input locations is similar to a fixed beam with a point load in between fixed supports. Hence the deflection will 
be maximum near the middle.For any input location, taper domain with point constraints give highest output displacement and the hexogonal domain with point constraints give the lowest output displacement. But the rectangular domain with point constraints gives the outputdispalcement values in-between.

\subsection{Effect of Output Locations $\left(\mathbf{X}_{0}\right)$}

To study the effect of output locations, the input force $\left(\mathrm{F}_{\mathrm{i}}\right)$, the input location $\mathrm{X}_{\mathrm{i}}=$ $\left(\mathrm{x}_{\mathrm{i}}\right) /\left(\mathrm{x}_{\mathrm{m}}\right)$ are kept constant. The output locations $\left(\mathrm{X}_{0}=\mathrm{x}_{0} / \mathrm{x}_{\mathrm{m}}\right)$ is varied as $0.083,0.167,0.25$, $0.33,0.417,0.5,0.583,0.667,0.75$ and 0.833 and the results are shown in the Figure 10.

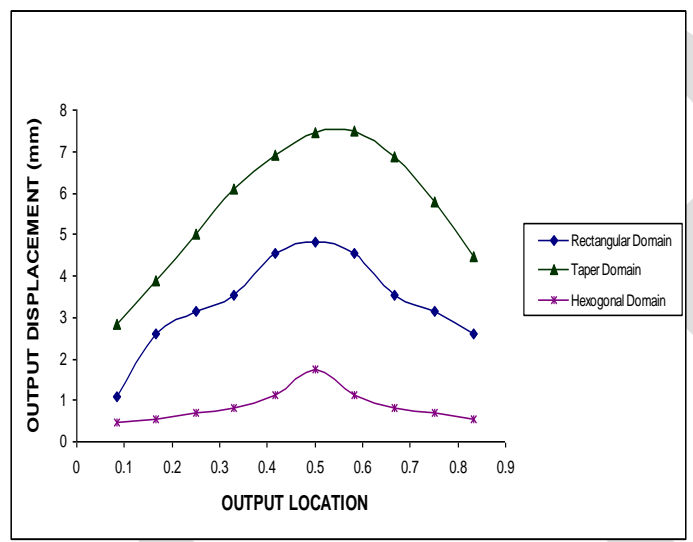

Figure 10 Effect of output locations $\left(X_{0}\right)$

The Figure 10 shows the maximum output displacement is obtained at $\left(\mathrm{X}_{\mathrm{o}}=\mathrm{X}_{\mathrm{o}} / \mathrm{X}_{\mathrm{m}}\right)$ is $7.48 \mathrm{~mm}$.

\subsection{Effect of Input Force $\left(\mathrm{F}_{\mathrm{i}}\right)$}

To study the effect of input force, the input location $\left(\mathrm{X}_{\mathrm{i}}=\mathrm{x}_{\mathrm{i}} / \mathrm{x}_{\mathrm{m}}\right)$, the output locations $\left(\mathrm{X}_{0}=\mathrm{x}_{0} / \mathrm{x}_{\mathrm{m}}\right)$ are kept constant. The magnitude of input force $\left(\mathrm{F}_{\mathrm{i}}\right)=100 \mathrm{~N}, 150 \mathrm{~N}, 200 \mathrm{~N}, 250 \mathrm{~N}$, $300 \mathrm{~N}$ and $350 \mathrm{~N}$ applied at $\left(\mathrm{X}_{\mathrm{i}}=\mathrm{x}_{\mathrm{i}} / \mathrm{x}_{\mathrm{m}}=0.5\right)$. The output displacement values obtained are shown in Figure 11.

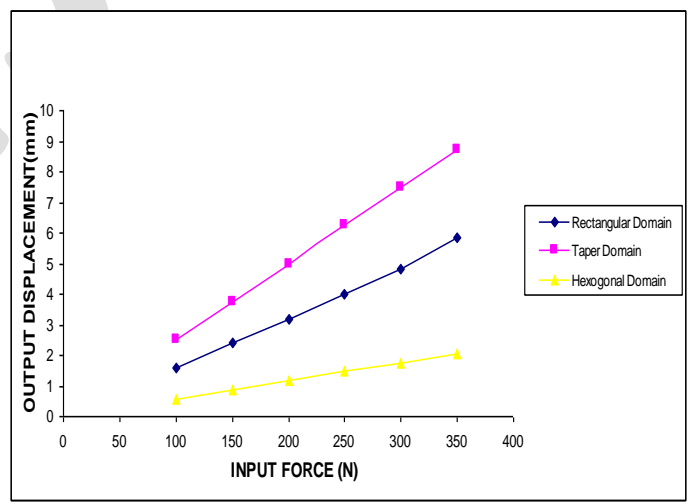

Figure 11 Effect of Input force $\left(F_{i}\right)$ 
The Figure 11 shows, while increasing the magnitude of forces applied at the input location the output displacement also increased linearly.

\subsection{Effect of Aspect Ratio (A)}

To study the effect of aspect ratio, the aspect ratio of the domain has been varied from $1.2,1.5,2,2.4 \& 3.0$. Figure 12 shows, when the aspect ratio increases, the output displacement are found to increase because the size of the domain decreases the output displacement will get increased.

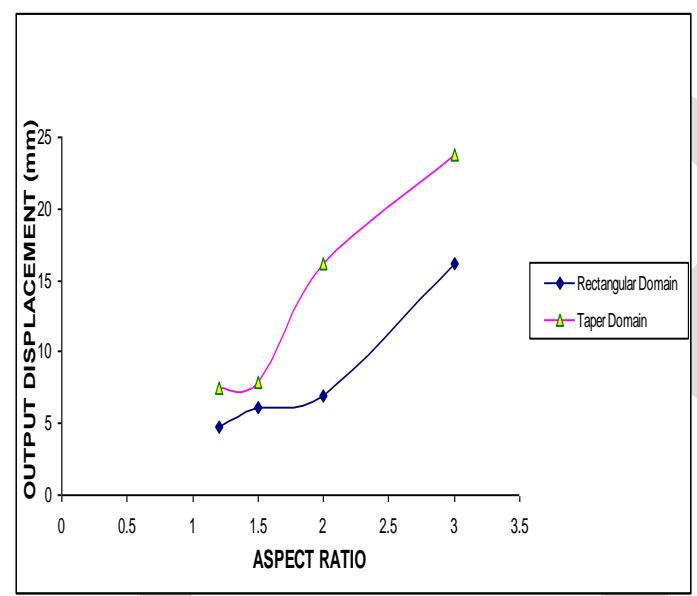

Figure 12 Effect of aspect ratio (A)

The Figure 12 shows, maximum output displacement is obtained, when the aspect ratio value is considered as 3 .

\subsection{Effect of Thickness (T)}

To study the effect of thickness, the input force $\left(\mathrm{F}_{\mathrm{i}}\right)$, the input location $\left(\mathrm{X}_{\mathrm{i}}=\mathrm{X}_{\mathrm{i}} / \mathrm{X}_{\mathrm{m}}\right)$ and the output locations $\left(\mathrm{X}_{0}=\mathrm{X}_{0} / \mathrm{X}_{\mathrm{m}}\right)$ are kept constant. The thickness of the domain is varied as $0.25 \mathrm{~mm}, 0.75 \mathrm{~mm}, 1 \mathrm{~mm}, 1.25 \mathrm{~mm}$ and the results of output displacement are shown in the Figure 13.

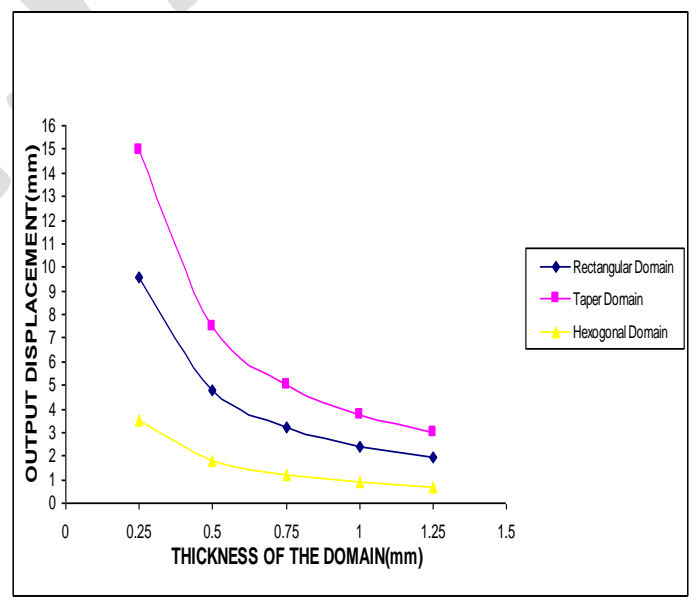

Figure 13 Effect of thickness 
Increase in domain thickness increases the cross sectional area of the element over which the input force is applied. The reduced stress results in lower input deflection in thickness of compliant mechanism reduce the output deflection for the given applied force.

\section{CONCLUSION}

In this work three regular shape of the design domains has been considered, however this method is applicable for any shape and size of the design domain. In the example problem the linear analysis has been considered, due to the requirement of topology optimisation and minimum displacement amplification at the output point.

To validate the numerical analysis results, experiment on compliant mechanisms and systems have been conducted and results have been checked.

The amplified displacement can be used for applications like opening and closing of proportional valves and vibrating a small chute etc.

\begin{tabular}{lll}
\multicolumn{2}{l}{ Symbols } & \\
$\mathrm{V}$ & - & Applied voltage \\
$\mathrm{U}$ & - & Deflection vector at a \\
$\mathrm{V}$ & - & Deflection vector at b \\
$\mathrm{fd}$ & - & Dummy unit force $(\mathrm{N})$ \\
$\mathrm{K}$ & - & Global stiffness matrix \\
$\Delta_{\text {in }}$ & - & Input deflection $(\mathrm{m})$ \\
$\mathrm{f}_{\mathrm{a}}$ & - & Input force $(\mathrm{N})$ \\
$\mathrm{a}$ & - & Input point \\
$\Delta_{\text {out }}$ & - & Output deflection $(\mathrm{m})$ \\
$\mathrm{b}$ & - & Output point \\
$\mathrm{A}$ & - & Vector of design variable
\end{tabular}

\section{ACKNOWLEDGEMENT}

The author acknowledges Sathyabama University for providing the facilities for the Numerical Experiments.

\section{REFERENCES}

[1].Howell L.L, "Design of Complaint Mechanisms" Willey Publications, pp 5-25, (2002).

[2]. Ananthasuresh G.K. and Anupam Saxena 'A Computational Approach to the Number of Synthesis of Linkages', Journal of Mechanical Design, Transactions of ASME, Vol. 125, pp. 110-118, (2003). 
[3]. Anandhasuresh G.K. and Saxena A., 'On an Optimal Property of Compliant Topologies', Journal of Structural Multidisc Optimization, Vol. 19, pp. 36-49, (2000).

[4]Howell LL and Midha A, " The Effects of a Compliant workforce on the input/output Characteristics of rigid link toggle mechanisms", Journal of Mechanism and Machine Theory, Vol.30, No.6, pp.801-810, (1995).

[5]. Arun Kumar, G, and Srinivasan, PSS, "Topology Optimisation of Complaint Mechanism for Displacement Amplification in Strain Actuators," IE (I)- Journal of Mechanical Engineering, Kolkatta, India, Vol. 86, pp.187-190, (2005).

[6]. Arun Kumar, and Srinivasan, PSS, "Design of Displacement Amplifying Complaint Mechanism with integrated Strain Actuator using Topology Optimisation," International Journal of Mechanical Engineering Science, SAGE Publishers, Vol.220, Issue 10, pp.1219-1228, (2006).

[7].Canfield S. and Fracker M., 'Topology Optimization of Compliant Mechanical Amplifiers', Journal of Structural Multidisc Optimization, Vol. 20, pp. 269-279, (2000).

[8] Fracker M and Kota S, "Topological Synthesis of Compliant Mechanisms Using Multicriteria Optimisation", Journal of Mechanical Design, Transaction of ASME, Vol.119, pp-238245, 1997.

[9]. Arunkumar, G.,Santhakumar, " Design of compliant mechanisms by topology optimization for strain actuators and engineering support ", International Review of Mechanical Engineering”, Vol.6, pp.979-989, 2012.

[10].Howell, LL and Midha, A, "A method for the Design of Compliant Mechanisms with Small-Length Flexural Pivots," ASME Journal of Mechanical Design, Vol.116, pp.280-290, 2012 .

[11]. Kota, S and Hetrick, J, "Synthesizing high-performance compliant stroke amplification systems for MEMS," Proceedings of the IEEE Micro Electro Mechanical systems, pp.164-169, (2000).

[12] Dong Xu and Anandthasuresh G K, "Freeform skeletal shape Optimization of Compliant Mechanisms", Journal of Mechanical Design, Vol.125, pp-253-261.

[13]Kota S and Rodgers SM, "Design of Compliant Mechanisms: Application to MEMS", Journal of Analog Integrated Circuits and Signal Processing, Vol.29, pp7-15, (2001)

[14]Lau G.K and Du H and Lim M.K), "Use of Functional specifications as Objective functions in Topological Optimisation of compliant mechanisms", Journal of Computer Methods in Applied Mechanics and Engineering, Vol.190, PP.4421-4433, 2001.

[15]Sigmond $\mathrm{O}$ and Torquato, "Design of Smart Composites Materials Using Topology Optimisation", Smart Materials and Structures, Vol.8, pp.365-379, (1999). 